TITLE. Reflected-Shock Initiation of Explosives

AUTHOR(S): Eric N. Ferm, LANL, M-8

Lawrence $M$. Hull, LANL, M-8

SUBMITTED TO: Tenth International Detonation Symposium

\section{DISCLAIMER}

This report was prepared as an account of work sponsored by an agency of the United States Government. Neither the United States Government nor any agency thereof, nor any of their employees, makes any wairanty, express or implied, or assumes any legal liability or responsibility for the accuracy, completeness, or usefulness of any information, apparatus, product, or process disclosed, or represents that its use would not infringe privately owned rights. Reference herein to any specific commercial product, process, or service by trade name, trademark, manufacturer, or otherwise does not necessarily constitute or imply its endorsement, recommendation, or favoring by the United States Government or any agency thereof. The views and opinions of authors expressed herein do not necessarily state or reflect those of the United States Government or any agency thereof. 


\title{
REFLECTED-SHOCK INITIATION OF EXPLOSIVES
}

\author{
E. N. Ferm and L. M. Hull
}

Explosives Technology and Applications Division

Los Alamos National Laboratory

Los Alamos, New Mexico 87545

\begin{abstract}
In a study of initiations caused by reflected shock from a high-impedance boundary attempts to establish sufficient conditions for initiation are described. Shock polar analysis is used to discover the ranges of various flow regimes. general shock structures and pressure estimates of states behind the reflected wave. Using this knowledge, wave structure growth rates from hydrocode simulations are estimated and standard-shock initiation criteria are used; experiments are designed in which the initiation from a reflected-shock wave structure appears likely. Two experiments are described in which a reflected-shock wave from a uranium surface initiated PBX 9502. The experimental evidence is in good agreement with the assumptions and results of the analysis.
\end{abstract}

\section{INTRODUCTION}

Shock initiation of explosives has been studied extensively and has resulted in useful models that describe the run to detonation, given the initial shock pressure. The experiments leading to these models were designed to eliminate boundary effects. The work presented here studies the influence of high-impedance boundaries on shock initiation of explosives. When boundaries are included in the problem, more analysis is required to determine the effect of wave reflections from the boundaries. Our approach to this problem was to examine the shock structure of the nonreactive reflected-wave problem using shock polar analysis and hydrocode simulations. We then looked for the shock structures that appear likely to initiate the explosive, considering the known explosive properties.

To obtain the shock structure, we studied the simple geometrical configuration of the reflection of a nonreactive, plane shock traveling in the explosive impacting a flat high-impedance metal plate like uranium. In this case, classical shock polar theory applies. This simple analysis determines the pressure behind the reflected wave as a function of the interaction angle and incident shock pressure. If the wave reflection is regular, the incident wave and the reflected wave are attached to the wall. If it is irregular, a more complicated shock structure must exist between the incident and reflected shock and the wall in order to satisfy boundary conditions. Shock polar analysis is only a local analysis, and it has limitations because the flow configurations are assumed in order to do the analysis. Our hydrocode calculations of the wave reflection problem were made to validate the shock polar analysis and to obtain growth rate information of the irregular reflection.
To find a case in which a reflected shock is likely to initiate an explosive, we expected the following conditions must be met: (1) the incident shock must not desensitize the explosive. ${ }^{1}(2)$ the amplified pressure must be sustained over the distances approximately equal to the single-shock run-to-detonation distance corresponding to the amplified pressure ${ }^{2}$ and (3) the pressure must be sustained over a width of explosive approximaiely the same as the failure radius of the explosive. ${ }^{3}$ These conditions are the same used on any shock to estimate whether initiation might occur; the only difference is that we are applying them to a local shock structure that occurs from a boundary interaction.

All the experimental work reported here studies the initiation of PBX 9502 by reflected waves from uranium boundaries. For PBX 9502, we found that irregular reflection was most likely to result in initiation. The primary reason is that the irregular reflection results in an amplified pressure wave running into explosive that was not preshocked. This wave structure, often referred to as a Mach stem or Mach reflection, is not the classical triple-point solution, but rather a curved-stem structure. We will discuss the analysis that suggests shock initiation with an irregular reflection or Mach reflection is possible, two experiments in which such initiation was detected for PBX 9502, and possible situations that may degrade or enhance the effect.

\section{CHAKACTERIZATION OF REFLECTIVE WAVE BEHAVIOR}

\section{LOCAL ANALYSIS}

The geometrical configuration we studied is a plane shock incident on a flat uranium plate. We applied the 
classical shock polar theory'. shown in Fig. I, to shock reflection in explosive. with approprate equations of state (see Table I).

When a shock wave obliquely encounters a highimpedance intertace. two basic kinds of shock reflection occur. Regular reflection is the case in which the incident wave and the reflected wave intersect at the intertace (Fig. 1a). Irregular reflection includes all other possibilities. of which there are many. The most tamous ir regular reflection is "classical" Mach retlection in which the interaction results in a triple-shock structure and a contact discontinuity separating the double-shocked material from the single-shocked material that has flowed through the stem structure isee Fig. Ibi. For brevity, any wave retlection structure that features a stem. where a single-shock processes material from zero pressure to the amplified pressure behind the retlection. will be called a Mach reflection.
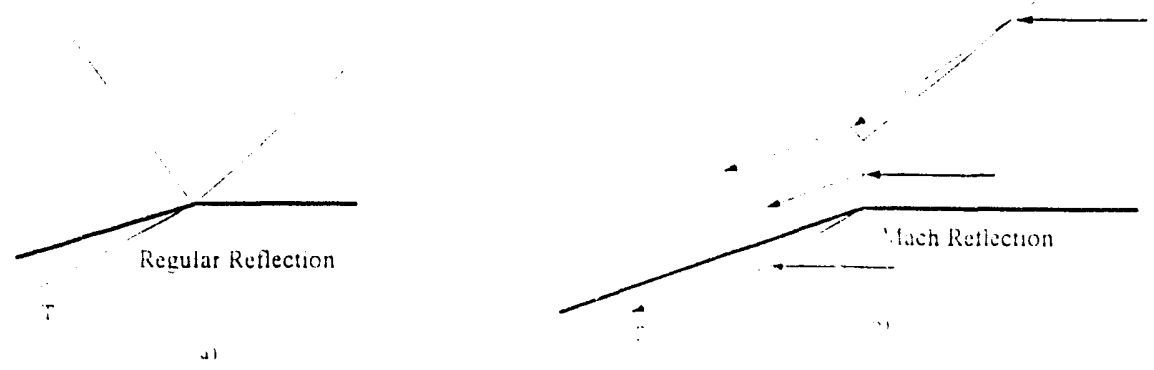

FIGURE 1. REGULAR AND MACH REFLECTION FLOW DIAGRAN

TABLE I. MIE.GRUNEISEN EQUATION-(OF.STATE PARAMETERS

\begin{tabular}{|l|c|c|c|}
\hline Material & $\begin{array}{c}\text { Density } \\
\left(\mathrm{g} / \mathrm{cm}^{3}\right)\end{array}$ & $\begin{array}{c}\text { Shock Hugoniot } \\
(\mathrm{mm} / \mathrm{us})\end{array}$ & $\begin{array}{c}\text { Gruneisen } \\
\text { Gamma }\end{array}$ \\
\hline PBX 9502 & 1.894 & $\mathrm{u}_{s}=2.36+2.23 \mathrm{u}_{n}$ & 1.2 \\
\hline Uranium & 18.93 & $u_{c}=2.51+1.51 u_{n}$ & 2.2 \\
\hline
\end{tabular}

In the local shock polat analysis, the flow in the regions delineated by the shocks is assumed to be one-dimensional. Shock jump conditions (conservation of mass. momentum, and energy subject to the equation of state) are satisfied across each shock. The incident shock turns the flow toward the wall and the reflected wave turns the flow back away from the wall. At the walls, the boundary conditions are such that the flow behind the shock structure must be parallel to the flow in the wall and pressure is continuous across the boundary. An irregular reflection appears when a regular reflection cannot meet this compatibility condition.

The shock polar in the pressure-flow angle plane is a convenient means of examining the possible solutions. Figure 2 indicates how the shock polar is generated: the phase velocity $V_{0}$ is fixed and the angle $\alpha$ between this velocity vector and the shock is varied. Jump conditions are used to calculate the normal component of the velocity behind the shock, and because the tangential component is unaltered, the flow angle can aiso be found. Flow angle $\theta$ and the pressure, the two variables of interest, are plotted in Fig. 3. The polar labeled $I$ indicates the states achievable behind the incident shock. The polar labeled $\boldsymbol{R}$ incicates the states achievable behind the reflected shock starting from the state behind the incident shock. The polar labeled $T$ indicates the states achievable behind the shock transmitted in the uranium. In regular reflection (see Fig. 3a), the flow behind the shock structure satisfies the boundary conditions at the intersection of the $T$ and $R$ polars. where the flow across the wall is parallel and no pressure gradient exists across the wall.

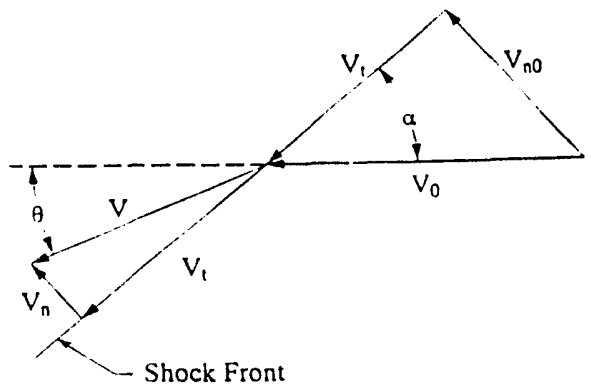

FIGURE 2. VELOCITY DECOMPOSITION OF 1-D FLOW ACROSS A SHOCK. 


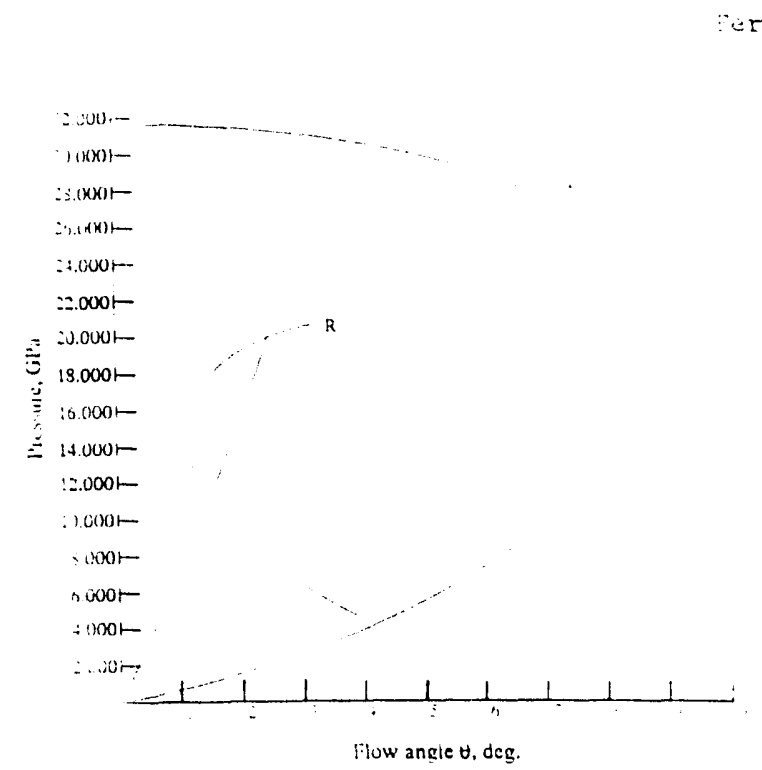

definition of the size of the Mach stem or its growth rate is lost. However. the shape of the $l$ polar indicates that some region of the flow will have reached amplified pressure levels through a single shock (the top of the thock polar is relatively tiat). Later, the wall pressure of this solution will be used as the stem pressure estimate.

Solutions to the retlection problem can also be plotted in the pressure-interaction angle plane. In this case. the wall pressure behind the retlected wave is given as a tunction of the interaction angle for tixed-incident shock pressure. That was done for a 42 -kbar incident shock in Fig. 4.

FIGURE 3a. SHOCK POLAR FOR REGULAR REFLECTION IN PBX 9502.

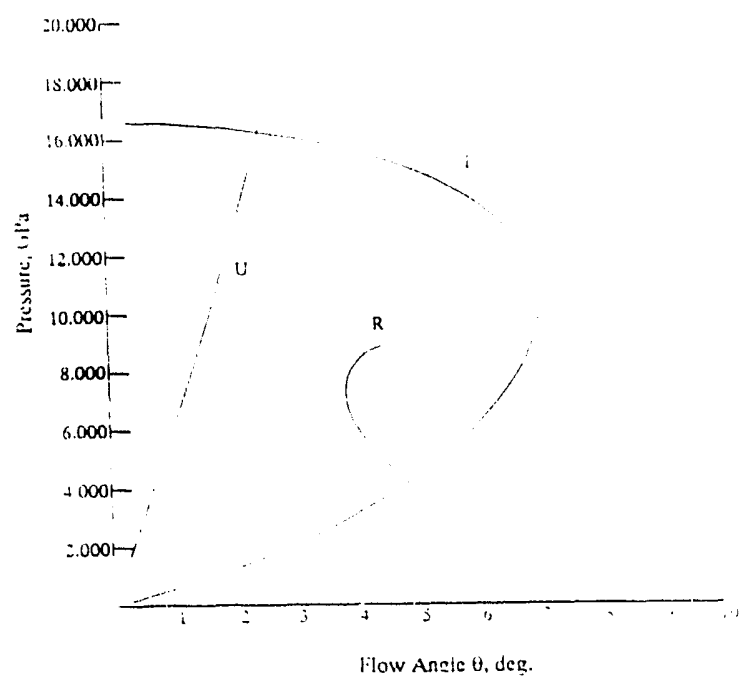

FIGURE 3b. SHOCK POLAR FOR IRREGULAR REFLECTION IN PBX 9502.

In Mach reflection (see Fig. 3b), the $T$ and $R$ polars do not intersect. If $\boldsymbol{I}$ and $\boldsymbol{R}$ had intersected at more than one point, a "classical" Mach reflection would be a possible solution. In this case, however, the incident shock will curve as the wall is approached. If the flow is quasi-steady, this analysis can be continued. This assumption is equivalent to assuming that the stem does not grow on a time scale of interest in these calculations. Continuing, we find that the flow behind the Mach stem at the wall is specified by the intersection of the $T$ and $I$ polars. Furthermore, the states on the curved Mach stem at positions displaced from the wall lie on the $I$ polar between the intersections of $\boldsymbol{I}$ with the $\boldsymbol{T}$ and $\boldsymbol{R}$ polars. Because of this continuous variation. the precise

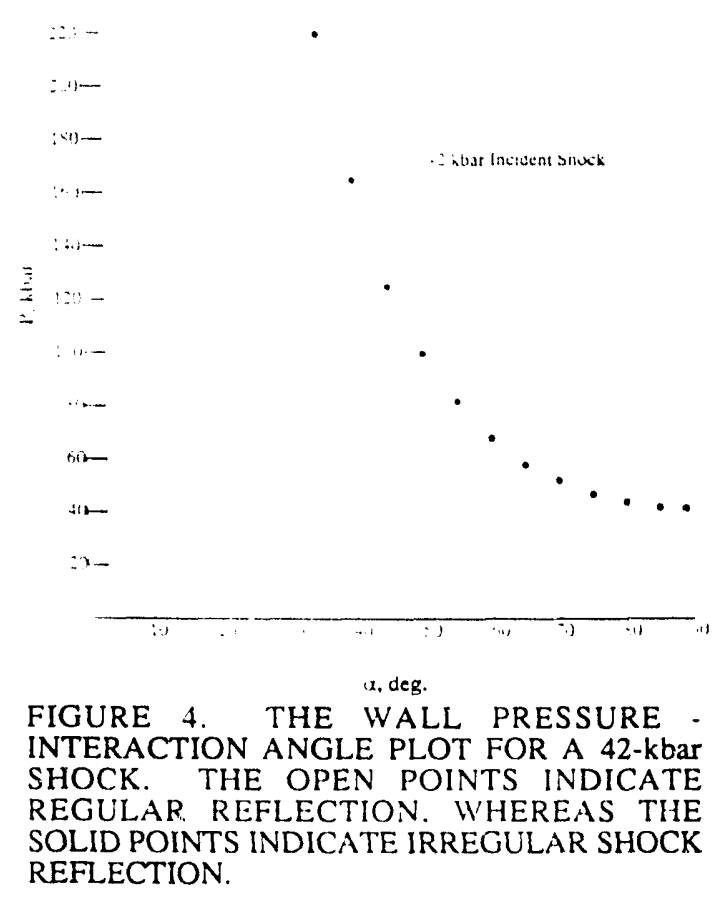

In either regular or Mach reflection, the pressure is elevated to a level that will initiate unshocked PBX 9502. In regular reflection, the elevated pressure propagates into material that has been preshocked and desensitized by the incident wave to some extent. In Mach reflection. however, the stem, with sufficiently elevated pressure behind it, propagates into unshocked explosive and represents a likely initiation mechanism. The shock polar analysis is convenient for giving critical angles and estimates of pressures; however, the analysis does not lead to any growth rate information, nor does it examine the stability of the flow configuration. To answer these questions, we examined this flow with the MESA hydrodynamics code.

\section{HYDROCODE ANALYSIS}

With MESA, we calculated several reflected-wave solution pressures and interaction angles, using a $0.5-\mathrm{mm}$ mesh. Shown in Figure 5 is the calculation of a 
42-kbar incident shock obliquely impacting a $j$-mm-thick uranium plate at $40^{\circ}$ incident angle $22 \mu \mathrm{s}$ atter the initial impact of the plate. Here a significant shock stem leads the incident shock. The 120 -kbar region backs a substantial portion of the stem and the 150 -kbar contour extends into the retlected-wave region. Even if the shock wave looks rather straight in this region, there is curvature in the shock wave and a more obvious continuous rise in shock pressure as the wall is approached.

Although these calculations are rather straight forward to complete and analyze. a subtle detail exists at the beginning of the calculation in how to resolve the initial point singularity on a finite mesh. In these cil. Mulations we took no special precautions. and if one looks carefully at the stem width in time, it appears to grow more rapidly at early times. then slows its growth significantly, and finally reaches an intermediate value. This appears to be the result of not being able to resolve the singularity at the beginning of the calculation. Hence, at eariy times in the calculation. the calculation appears to overestimate the stem width and underestimate the pressure. This problem appears to resolve itself in time. However. some caution should be used in the examination of early results from the simulations.

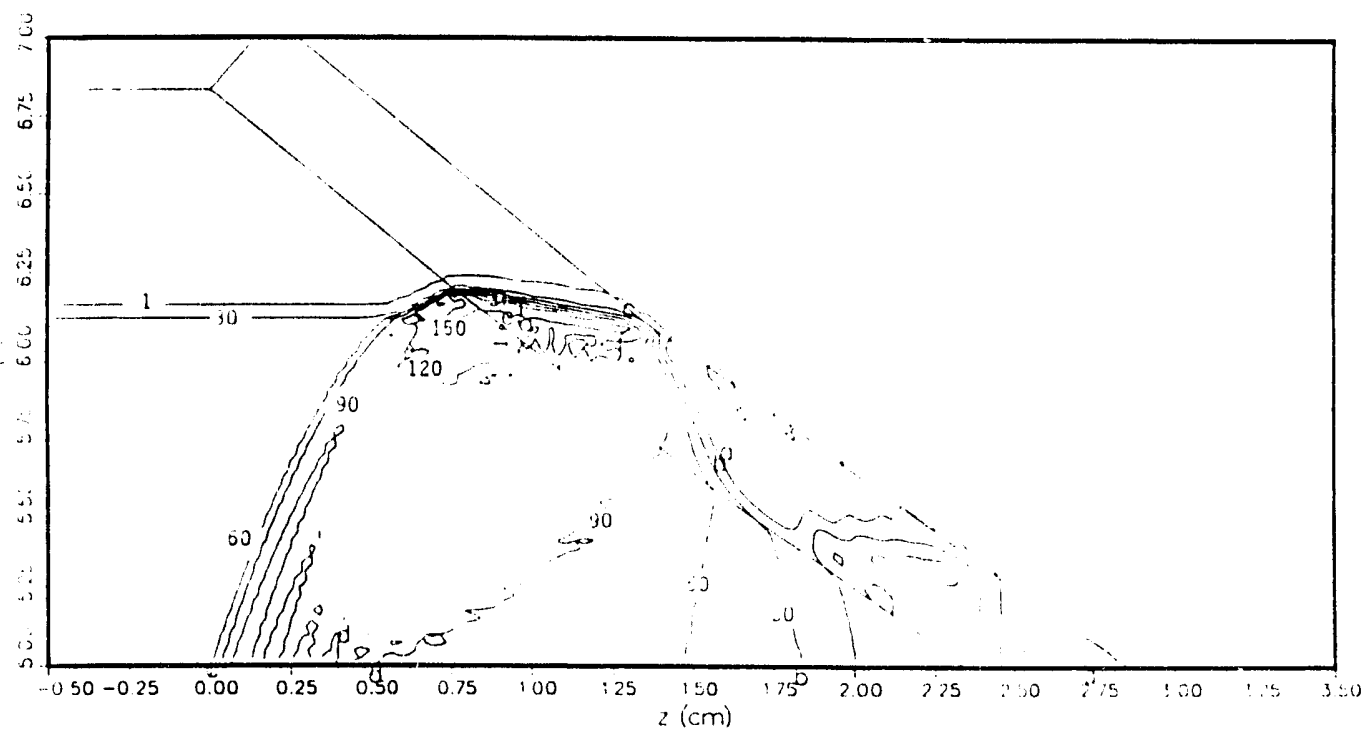

FIGURE 5. MESA CALCULATION OF A 42-kbar SHOCK INCIDENT ON A 3-mm URANIUM PLATE. PRESSURE CONTOURS ARE LABELED IN KILOBARS.

In summary, the hydrocode simulations are in qualitative agreement with the shock polar analysis, although the pressure values from the hydrocode tend to have somewhat lower pressures than the estimates from shock polar theory. The growth angle of the stem region found in these calculations ranges from $1^{\circ}$ to $5^{\circ}$, depending on the incident shock pressure and angle. The small growth angles can be used to rationalize the use of the quasisteady approximation in the previous section. This range of angles is typical for growth rates of Mach stems seen in gas dynamics 5 and detonation experiments. 6

\section{INITLATION ANALYSIS}

The preceding analysis has shown that in either regular or irregular reflection, the pressure amplification is sufficiently large to dramaticaily reduce the distance to detonation of unshocked explosive. ${ }^{2}$ In considering the possibility of initiating by a regular reflection, many casss can be ruled out because the flow behind the second shock is highly supersonic, and any energy release in the double-shocked material hiss no possibility of influencing either the reflected or the incident shock. As the incidence angle approaches the critical angle, this argument cannot be used because the flow behind the, reflected shock becomes subsonic. In these cases, consider the thickness of material that can travel throu gh the incident shock and reach the second shock before it has been desensitized by the incident shock. This width can be estimated by assuming the time that the material is in the incident-shock region must be less than the time it takes for the explosive to be desensitized. We used the time estimate from Campbell and Travis's paper ${ }^{1}$ for PBX 9404, because no desensitization data are available for PBX 9502. For a 50-kbar shock at $25^{\circ}$ incidence angle, the width of material in the second shock region conforming to this criterion is $0.5 \mathrm{~mm}$, which is much smaller than the self-confined failure thickness, and even smaller than the run-to-detonation distance for the pressure in the double-shocked explosive region. This material may react, but it has low probability of developing into a detonation because it is significantly smaller than the failure radius. ${ }^{3}$ A more positive statement is precluded because the self-confined failure radius of the 
precompressed explosive has never been studied. This initiation scenario may be much more important in more sensitive explosives like PBX 9501. These have much smaller failure thicknesses than do insensitive explosives, and much lower incident shock pressures result in much larger critical angles. In all. we were led to examine the Mach reflection regime, where preshock was not an issue.

In Mach reflection, with sufficiently elevated pressure behind it, the stem propagates into unshocked explosive and presents a likely initiation case because the explosive has not been desensitized. Another aspect of Mach reflection enhances the likelihood of a situation in which initiation can occur: the flow behind a large portion of the curved shock wave section is subsonic. This implies that energy release from the shocked explosive has a chance of reinforcing the shock wave and of building to a detonation wave.

The remaining requirement is that the Mach stem must be sufficiently large. Classical shock polar analysis provides no stem growth information in this curved-stem case. However, the hydrocode calculations indicated a growth angle of $1^{\circ}$ to $5^{\circ}$. This would imply that initiation would occur after a run of 44 to $229 \mathrm{~mm}$ along the surface of the uranium plate, depending on the shock pressure and angle. Using this analysis, we designed the experiments to detect the transition from shock to detonation when a 40-kbar plane shock in PBX 9502 is reflected from a tlat uranium plate with an incidence angle of $40^{\circ}$ and $50^{\circ}$.

\section{EXPERIMENTAL RESULTS}

The PBX-9502 test pieces were six-sided prisms (see Fig. 6). Top and bottom surfaces defined two horizontal planes $96.4 \mathrm{~mm}$ apart. Two sides defined planes that were perpendicular to the top and bottom planes. One of the other two surtaces ( $A$ in Fig. 6 ) was angled at $30^{\circ}$ to the bottom plane and was defined as the observation surface. The other surface (B in Fig. 6) was angled at $40^{\circ}$ or $50^{\circ}$ with respect to the bottom plane and was covered with a 3 -mm-thick uranium plate providing the reflective boundary. A plane shock wave was driven into the bottom of the prism with the plane wave attenuator system shown in Fig. 7.

The phase velocity of the wave along the uranium surface, the width of the Mach stem, and the incident wave velocity, pressure, and position must be observed to obtain a complete experimental record of the experiment. The shock arrival time along the uranium surface (B) was monitored with a series of time-of-arrival pins that we placed at known distances along the uranium plate. Each pin reported the time at which the shock transmitted (at some angle) through the uranium plate ar rived at the free surface. We estimated the phase velocity of the wave at the explosive-metal interface from these data by assuming the flow to be steady. We obtained the width, shape, and velocity of the Mach stem, as well as the incident v:ave velocity by using a multiple-slit smear camera technique. The intersection of surfaces $A$ and $B$ and at least $25 \mathrm{~mm}$ of surface $A$ were observed with a series of argon flash gaps. Each camera slit was aligned so that a record of the flash gave the wave arrival time of the Mach stem and incident shock at 11 different run distances into the explosive.

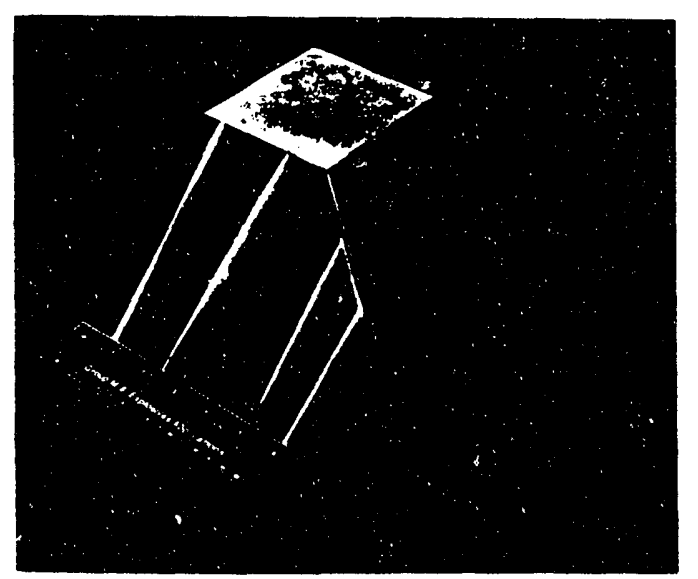

FIGURE 6. PBX-9502 TEST PIECE WITH URANIUM PLATES ATTACHED.

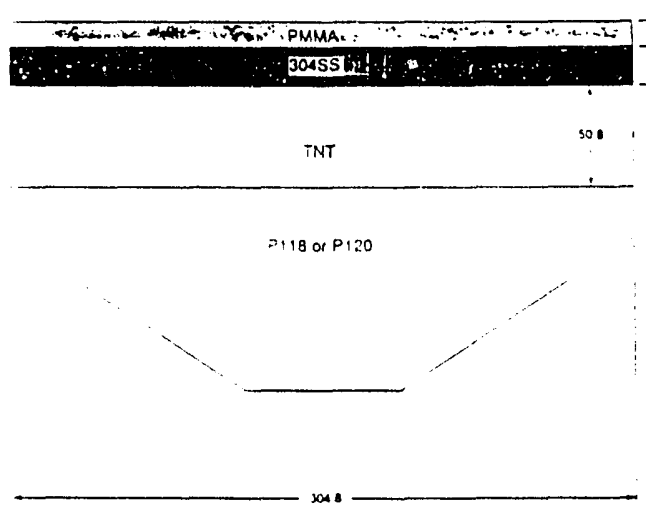

FIGURE 7. THE PLANE SHOCK DRIVER SYSTEM USED TO DRIVE A $+2-k b a r$ SHOCK INTO THE TEST PIECE OF PBX 9502. DIMENSIONS ARE IN MILLIMETERS.

Shown in Fig. 8 are the shock arrival times on the uranium surface for the $40^{\circ}$ experiment. Straight-line fits show that the incident phase velocity associated with the propagation of the inert Mach reflection is about 6.02 $\mathrm{mm} / \mu \mathrm{s}$. About $75 \mathrm{~mm}$ from the corner, a transition to $7.43 \mathrm{~mm} / \mu \mathrm{s}$ occurs. Similar results for the $50^{\circ}$ interaction angle, also shown in Fig. 8, indicate that the wave accelerates from $4.92 \mathrm{~mm} / \mu \mathrm{s}$ to $7.68 \mathrm{~mm} / \mu \mathrm{s}$ after transiting the surface for $70 \mathrm{~mm}$.

In Fig. 9 is the digitized camera record from the $40^{\circ}$ shot, and the smear camera record of the $50^{\circ}$ experiment is shown in Fig. 10. Each slit was oriented so that a record of the wave arrival time was obtained for a constant distance from the bottom surface of the prism. Each slit recorded the arrival of the incident wave as a 
horizontal line in the region away irom the uranium interface. These were used to obtain the velocity of the incident shock and thus to estimate the incident pressure The Mach stem developed at the uranium-PBX-950) intertace was recorded as the early-arrival part of each trace. When the location of the break in the horizontal trace was measured, the width of the Mach stem or detonation front could be estimated as a function of run distance along the uranium plate. Assuming that the Mach stem is normal to the wall. a conservative estimate for the Mach stem width can be obtained. The assumption that the Mach stem is normal to the wall may be relaxed if one is willing to accept a calculated value for this angle. Mach stem width data are plotted as a function of position along the uranium plate in Fig. 11. A sudden change in wave width is associated with the transition to detonation. This is in good agreement with the previously described pin data. The last slit recorded a detonation wave $7 \mathrm{~mm}$ wide in the $40^{\circ}$ case and $17 \mathrm{~mm}$ wide in the $50^{\circ}$ case.

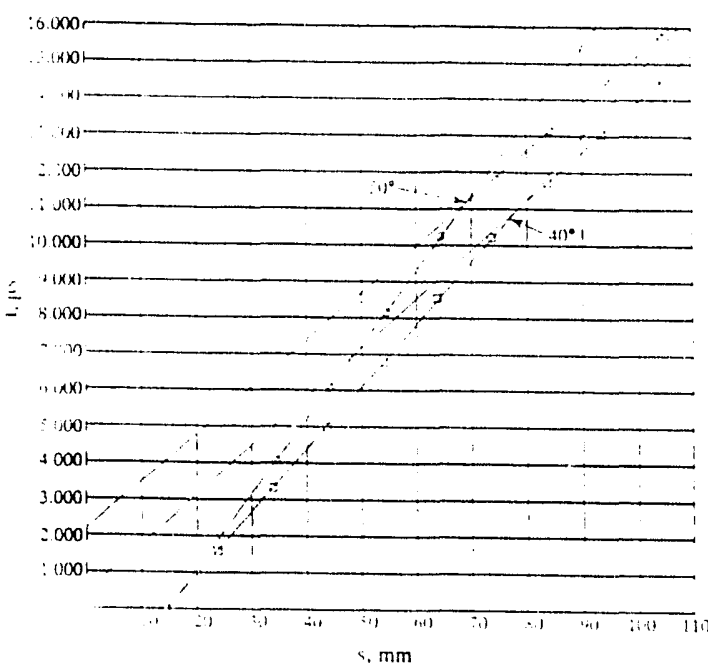

FIGURE 8. PIN DATA FOR SHOT Nos. C-6496 AND C-6543

The two smear camera records clearly show the de velopment of curved Mach stems with growth angles of $1.7^{\circ}$ and $3.6^{\circ}$. The transition occurred when the stem reached a width between 2 and $4 \mathrm{~mm}$ in the $40^{\circ}$ case and between 4 and $6 \mathrm{~mm}$ in the $50^{\circ}$ case. After the transition, a detonation wave is clearly observed with a growth angle of more than $5^{\circ}$ in the $40^{\circ}$ incident angle case and more than $15^{\circ}$ in the $50^{\circ}$ case. We expect that this angle is strongly influenced by corner-turning and preshock-desensitization processes. These runs to detonation, measured from the driver system, are 1/5 of what one would expect from extrapolating existing run-to-detonation data to the incident shock pressure.

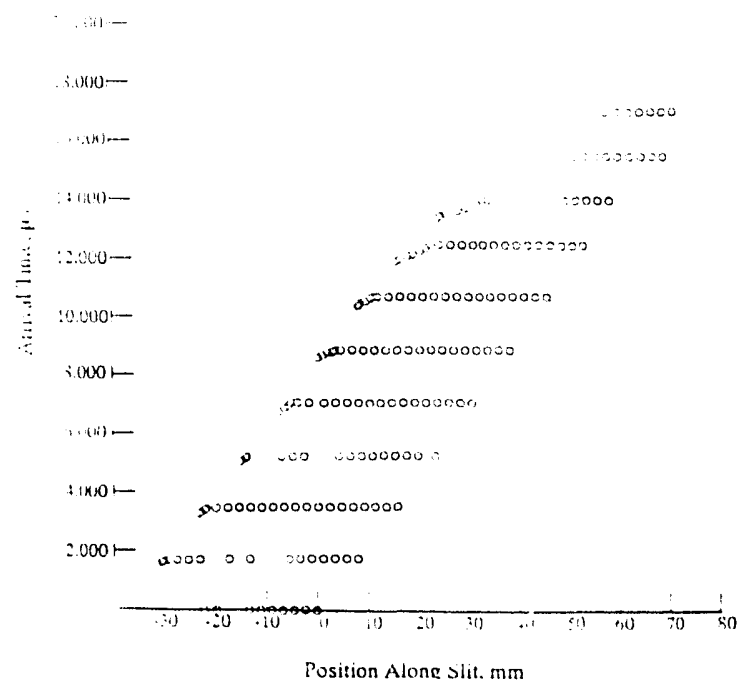

FIGURE 9. DIGITIZED SMEAR CAMERA RECORD FOR SHOT No. C-6496, INCIDENT SHOCK WAS 42 Lbar AT 40 INCIDENCE.

\section{DISCUSSION}

These experiments demonstrate that in PBX 9502. a Mach reflection can transit into a detonation for two different incident angles. Diameter effect data ${ }^{3}$ indicate that a 7-mm-radius rate stick of PBX 9502 would detonate at $7.49 \mathrm{~mm} / \mu \mathrm{s}$ and a $17 \cdot \mathrm{mm}$-radius rate stick detonates at $7.62 \mathrm{~mm} / \mu \mathrm{s}$. The final pin velocities agree well with these values. Mirimum width of the detonation observed is about $4 \mathrm{~mm}$, and this is close to the failure radius of $4.5 \mathrm{~mm}$ for PBX 9502.3 Pressure behind the Mach stem was estimated at $160 \mathrm{kbar}$ for $40^{\circ}$ and $100 \mathrm{kbar}$ at 5()$^{\circ}$. based on the shock polar theory. Runto-detonation distance for a planar. well-suppored shock at these pressure is about $4 \mathrm{~mm}$ and $13 \mathrm{~mm}$, respectively. ${ }^{4}$ Our experiments sustained the shock pressures much longer than this. However, the flow behind the stem shock is substantially more complicated than that found in a wedge experiment. Therefore, the conditions we detected are in agreement with our expectations derived from other aspects of explosive behavior. Because the shock initiation estimates of where initiation occurs appear to hold, one would expect a multiple-shock Forest Fire model to be able to model these problems adequately. Calculations ${ }^{6}$ done by Ed Kober show that this is indeed true and give extra evidence that the initiations in these cases are due to the shock-reflection structure.

Further work continues on this topic in the areas of other explosive materials, divergence effects of the incident shock wave, and the effect of convergent and divergent wall interfaces. We are using PBX 9501 to assess the implications of this initiation mechanism on much more sensitive explosives. Classical analysis indicates that for PBX 9501, the angles at which Mach stems become important are about $20^{\circ}$ greater than those of PBX 9502. primarily because the pressures at which initiation 
from a plane shock occurs are significantly smailer Although the range of possible angles is smaller. the initiation appears likely because the failure diameter is much smaller and the run to detonation is significanty shomer than for that of PBX 9502. Also, for PBX 9501. some cases of initiation by regular reflection may be possible.

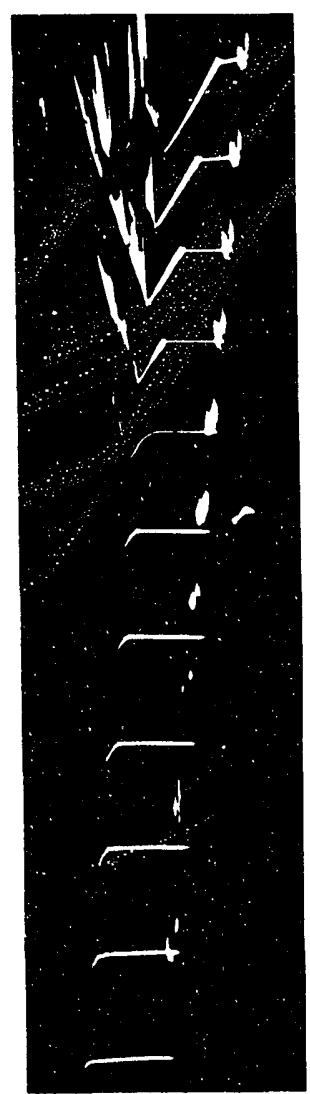

FIGURE 10. SMEAR CAMERA RECORD FOR SHOT No. C-6543. INCIDENT SHOCK WAS 42 kbar AT $50^{\circ}$ INCIDENCE. FOR EACH SLIT, TIME IS INCREASING IN THE VERTICAL DIRECTION. THE OVEREXPOSURE ON THE STEM IN THE LAST FOUR SLITS IS DUE TO THE BRIGHT FLASH RESULTING FROM THE DETONATION, AS COMPARED WITH THE EXPOSURE OF THE INCIDENT SHOCK.

Divergence can occur in two different ways: the incident shock can diverge or the metal boundary can curve away from the incident shock. In both of these cases, computer simulations show that the formation of the Mach stems is inhibited. Experiments are currently being designed to examine these effects. We have only begun to study convergent geometries that we expect will enhance the formation of Mach stems.

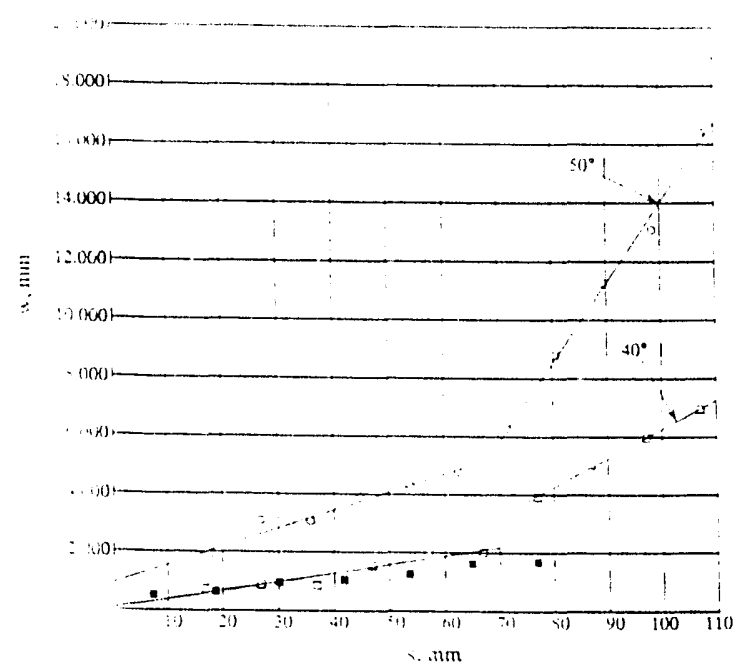

FIGURE 11. STEM WIDTH MEASUREMENTS FOR SHOT Nos. C-6496 AND C-6543. THE SOLID SQUARES ARE STEM WIDTHS MEASURED FROM TIIE MESA CALCULATION OF THE REFLECTION OF A +2-kbar SHOCK INCIDENT AT $41^{\circ} \mathrm{ON}$ A URANIUM SURFACE.

In summary, we have experimentally observed the transition of a Mach reflection into a detonation in PBX 9502. This initiation mechanism can radically reduce the run to detonation in explosive charges with high-impedance boundaries. Classical analysis can bound the incident shock pressures and angles of Mach reflection initiation. The resulting shock contigurations and knowledge of explosive behavior can be used to help decide whether initiation is likely. However, growth angle and stem size information is not found by means of the classical analysis. Computer simulations are useful for obtaining growth angle estimates and Forest Fire models can be used to examine the initiation process. Even so, there are still mesh resolution problems to address, and equation of state (especially sensitive materials) information for the explosive at low pressures will need to be refined before simulations are reliable. Therefore, further experiments and analysis will be necessary to completely describe the initiation criterion for shock reflection from high-impedance boundaries.

\section{ACKNOWLEDGMENTS}

We express our appreciation to Victor Sandoval, Willie Spencer, Rudy Archuleta, Bob Critchfield, Andreas Krause, and Walter Quintana. who were responsible for, or assisted in, firing these experiments. We thank Ed Kober for discussing with us his Forest Fire simulations of initiation by shock-wave reflection. We acknowledge that LeRoy Green had the insight to suggest the possibility of initiation by a Mach stem. We thank John Ramsay for many helpful discussions and support. We also thank Larry Hantel and John Ramsay for their persistent support in obtaining funding. 

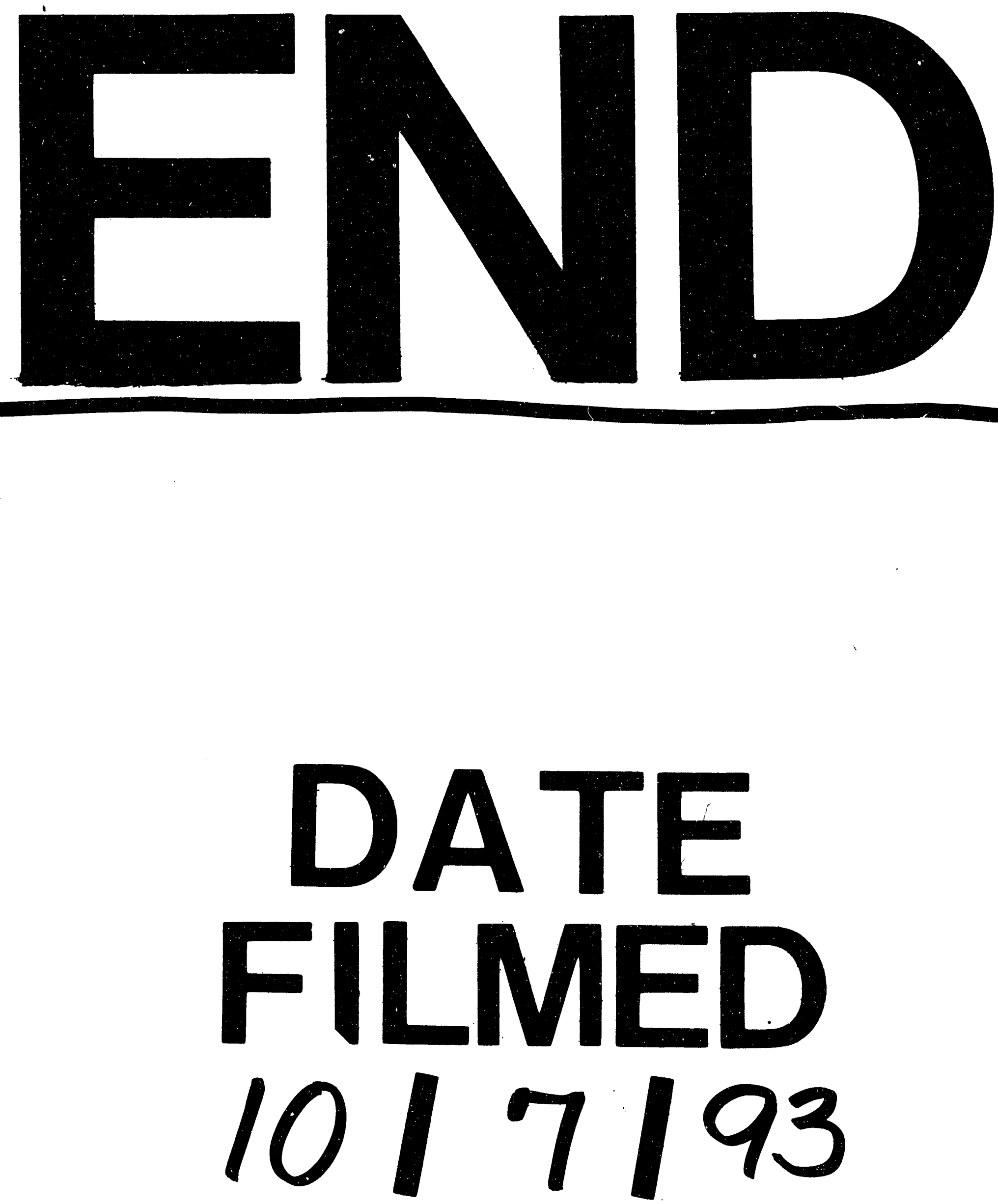


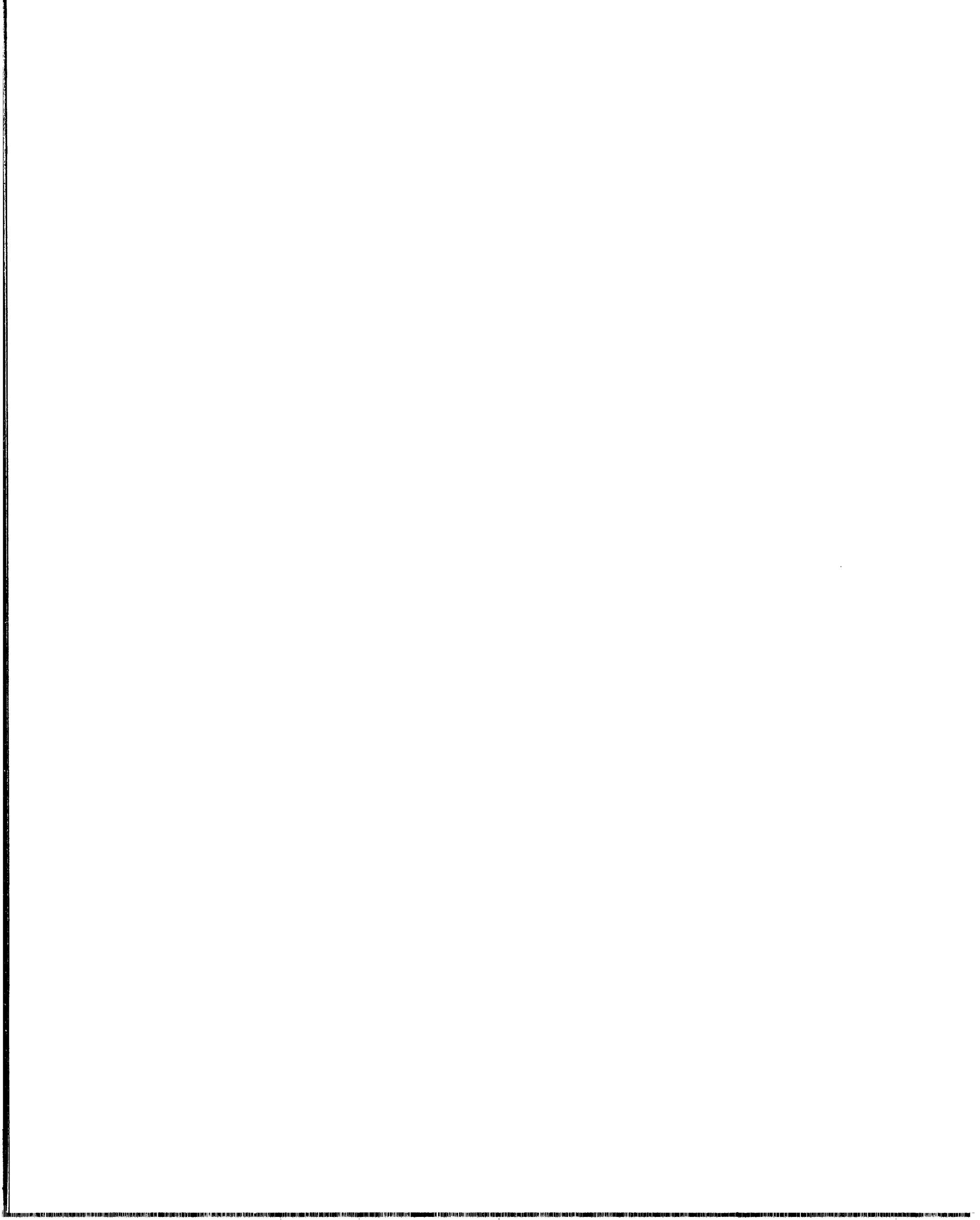

